\title{
Applying Mobile Agent to Intrusion Response for Ad Hoc Networks
}

\author{
Ping Yi, Yiping Zhong, and Shiyong Zhang \\ Department of Computing and Information Technology Fudan University, 200433, China
}

\begin{abstract}
Mobile ad hoc networking offers convenient infrastructureless communication over the shared wireless channel. However, the nature of ad hoc networks makes them vulnerable to security attacks. Existing security technologies such as intrusion prevention and intrusion detection are passive in response to intrusions in that their countermeasures are only to protect the networks and there is no automated, network-wide counteraction against detected intrusions. While they all play an important role in counteracting intrusion, they do not, however, effectively address the root cause of the problem - intruders. In this paper, we propose the architecture of automated intrusion response. When an intruder is found in our architecture, the block agents will get to the neighbor nodes of the intruder and formed the mobile firewall to isolate the intruder.
\end{abstract}

\section{Introduction}

Mobile Ad hoc Network is an autonomous system of mobile nodes connected by wireless links. Each node operates not only as an end-system, but also as a router to forward packets. The nodes are free to move about and organize themselves into a network. Mobile ad hoc networks does not require any fixed infrastructure such as base stations, therefore, it is an attractive networking option for connecting mobile devices quickly and spontaneously, such as military applications, emergent operations, personal electronic device networking, and civilian applications like an ad-hoc meeting or an ad-hoc classroom.

With more and more application, security for mobile ad hoc networks becomes increasingly important. Existing security approaches for networks can be categorized as intrusion prevention, intrusion detection and intrusion response. Intrusion prevention utilizes authentication, encryption and firewall etc. to protect system from being attacked and compromised. However, some way may not be applied to mobile ad hoc networks, such as firewall. Because of dynamic topology, mobile ad hoc networks do not have a clear line of defence. When nodes roam in a hostile environment with relatively poor physical protection, they have probability of being compromised. The compromised nodes may launch attacks within the networks. Encryption and authentication can not defend against compromised nodes, which carry the private keys. Therefore, these approaches of intrusion prevention which can play an important role in traditional networks may take limited affection in mobile ad hoc networks.

Intrusion Detection Systems (IDS) attempts to detect intrusion by analyzing observed system or network activities. The IDS may be classified based on the detection technique, such as anomaly detection, misuse detection and specification-based detection. IDS will raise alarms when it has detected an attack. 
While intrusion prevention and detection all play an important role in solving the problem of today's network-based intrusion, they are all passive and not adequate to solve the intrusion problem. One fundamental problem with existing intrusion prevention, detection and tolerance is that they do not effectively eliminate or deter networkbased intrusions. The best they can do is to avoid being victims of network-based intrusions temporarily. Because they do not address the root cause of intrusions intruders, those intruders can always explore new system vulnerabilities, find new accomplices - potentially insiders, and launch new attacks from different places. Because intrusion prevention and detection do not effectively address the problem of compromised system recovery, intruders can use those compromised system as new base for further intrusion. What we need is an effective way to hold network-based intruders accountable for their intrusions, and an automated way to prevent compromised hosts from doing further harm.

So far most computer security research regarding intrusions has focused on prevention and detection. Intrusion response has been an afterthought and is generally limited to logging, notification. There are more vulnerability in mobile ad hoc networks for its wireless link and mobile node. If no response as soon as early, intruder may destroy entire networks. In addition, for self-organizing and lack of centralized administration, manual response is difficult to bring into effect, especial in multiple administrative domains.

In this paper, we propose Mobile Firewall to address the problem of intrusion. Our goal is to effectively and rapidly eliminate intrusion and reduce the damage as soon as possible by means of automated intrusion response. We make three contributions to the area of intrusion response for mobile ad hoc networks.

- Mobile Firewall: In a traditional network, a firewall is installed at the ingress point of the network to filter out unallowable traffic originating from outside the boundary of the protected network. In the paper, the Mobile Firewall is used to surround and isolate the intruder. The Mobile Firewall can move with the intruder node and isolate the intruder for ever.

- Shrapnel way to dispose: An artillery shell containing metal balls fused to explode in the air above enemy troops. By analogy, agent moves to the node around the intruder and reproduces a lot of agents to surround and isolate the intruder in the end.

- Local repair: when the intruder is found, all paths via the intruder will find a new route to bypass the intruder by the process of local repair.

The rest of the paper is organized as follows. Section 2 gives the related work. Section 3 addresses the approach of automated intrusion response. And section 4 concludes the paper.

\section{Related Work}

The Boeing Phantom Works carries out some researches in autonomic intrusion response. D. Schnackenberg et al present the Cooperative Intrusion Traceback and Response Architecture (CITRA) who integrated networks-based intrusion detection systems, firewalls, and routers to trace attacks back to their true source and block the 
attacks close to that source [1]. D. Schnackenberg et al provide an overview of the Intruder Detection and Isolation Protocol (IDIP) [2]. Dan Sterne et al present the results of testbed experiments using CITRA and IDIP to defend distributed denial of service attacks [3]. The CITRA and IDIP support automatically tracing attempted intrusions across network boundaries and blocking or otherwise responding to them near their sources. This technology will enable networks of networks to cooperatively detect system attacks, exchange information about attack behaviour, and respond to attacks by dynamically reconfiguring routers, firewalls, and hosts to heighten their defensive posture and provide additional protection against subsequent attacks. The heart of this technology is a new protocol, the Intruder Detection and Isolation Protocol (IDIP), which enables cooperation among such components. Working together in internet worked systems, IDIP-enabled components can locate, isolate, and block an intruder close to the point of attack and provide diagnostic information so that network administrators can further investigate the intrusion. The above technology can take effect in traditional wireline networks environment, but they are not applied to mobile ad hoc networks for the wireless link and dynamic topology.

X. Wang et al propose Tracing Based Active Intrusion Response (TBAIR) [4]. Based on Sleep Watermark Tracing (SWT), TBAIR can trace the detected intrusion and push the intrusion countermeasures close to the source of the intrusion. N. Foukia et al present an approach inspired by natural systems for intrusion detection and response [5]. Its approach uses the immune system metaphor for intrusion detection agents and social insect's behavior metaphor for intrusion response. CA Carver et al propose a methodology for adaptive intrusion response using intelligent agents [6]. Jansen $\mathrm{W}$ et al propose a number of innovative ways to apply agent to address the shortcomings of current intrusion detection system [7]. C. A. Carver, and U. W. Pooch provide a categorization of possible offensive and defensive responses.

The papers of mobile ad hoc networks security can be classified in three categories: key management, secure network routing, and intrusion detection. Capkun, Buttyan and Hubaux propose a fully self-organized public key management system that can be used to support security of ad hoc network routing protocols [8]. Zhou and Hass first proposed to use threshold cryptography to securely distribute the Certificate Authority private key over multiple nodes to form a collective CA service[9]. Routing security has been most noted by its absence early in the discussion and research on ad hoc routing protocols. Since then several ad hoc routing protocols that include some security services have been proposed: SRP[10], Ariadne[11], ARAN[12], SEAD[13]. SRP[10] assumes the existence of shared secrets between all pairs of communicating nodes and leverages this for MAC authentication, such that fake route requests are not accepted at the destination and routes set in route replies cannot be modified. In Ariadne[11], end-to-end authentications are got by one-way hash chain and MAC authentication. ARAN[12] relies on public key certificates to retain hop-by-hop authentications. SEAD[13] use elements from a one-way hash chain to provide authentication for both the sequence number and the metric in each entry. Yongguang Zhang developed an Intrusion Detection architecture and evaluated a key mechanism in this architecture, anomaly detection for mobile ad-hoc networks [14]. These security systems only passively detect and prevent invader, they can not effectively eliminate or deter intrusions. 


\section{The Approach of Automated Intrusion Response}

\subsection{System Architecture}

An agent is a small intelligent active object which is able to carry out activities continuously and autonomously in a particular environment. Agent is autonomous, lightweight, adaptive, and mobile. Multi-agent can communicate and cooperate with each other. These qualities make agent a choice for security architecture in bandwidth and computation-sensitive mobile ad hoc networks.

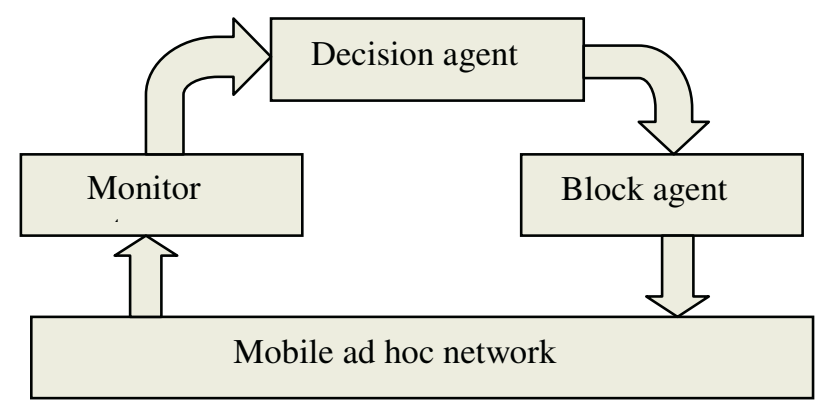

Fig. 1. System architecture

Figure 1 shows the three essential components of the architecture: monitor agent, decision agent and block agent. Monitor agent resides on each node and monitors the neighbour nodes by collecting all packets with its communication range. Monitor agent code behaviour information of its neighbour nodes and send them to decision agent. Decision agents collect the information from the monitor agent, and make a judgment by security policies. If the decision agent can judge that some node is invader, it will produce block agents. These block agents move to the neighbour nodes of the intruder and construct Mobile Firewall to surround the invader and isolate the intruder in the end. The whole response process is automated without manual intervention.

\subsection{Mobile Firewall}

In a traditional network environment, a firewall is installed at the ingress point of the network to filter out unallowable traffic originating from outside the boundary of the protected network. In mobile ad hoc wireless environment where the nodes could be mobile and topology of networks is dynamic, there is therefore no notion of a well defined ingress point for the network. In addition, any node within the network could become an intruder for compromised and so attack traffic could originate from within the network itself. Hence, the traditional firewall concept does not work in an ad hoc network. Furthermore, traditional firewalls are not designed to protect against spoofed packet flooding attacks where the attack traffic masquerades as legitimate packets passed through by the firewall access control rules. 
The Mobile Firewall is used to protect mobile ad hoc networks against attacks launched from an intruder node within the networks. Mobile ad hoc networks are multi-hop wireless networks, and the node sends and receives packets through its neighbor nodes. If all neighbor nodes around the node refuse to forward its packets, the node can not communicate with the other nodes in mobile ad hoc networks. The node has been isolated from the network in practice even if it is still in the networks in location. The Mobile Firewall takes effect using the above principle. When decision agent finds an intruder, decision agent sends block agents to the neighbor nodes of intruder. These block agents reside in the nodes around the intruder and surround the intruder. In the mean time, they cut off the links between the nodes and the intruder, and they do not receive and forward any packet from the intruder. In the end the intruder is isolated from network. The traditional firewall is to protect the networks in firewall against outside attack, and the Mobile Firewall is to surround the intruder and protects the other nodes in network. The Mobile Firewall can block an intruder close to the point of attack. It may be more efficient and more secure.
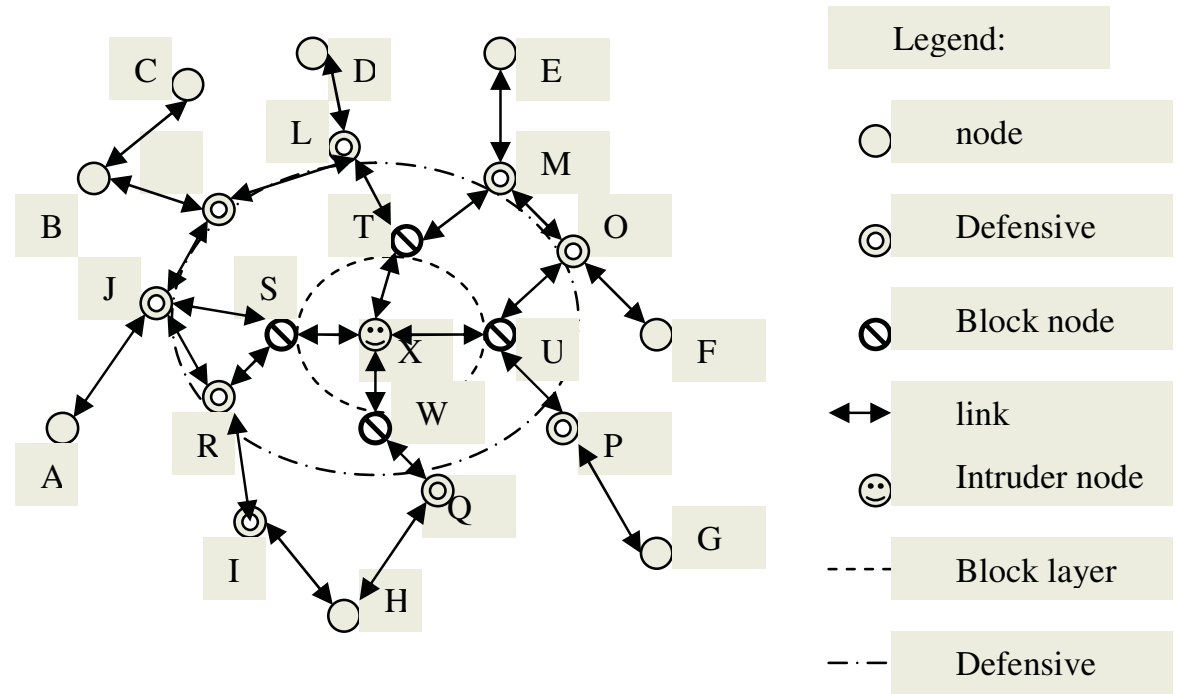

Fig. 2. The Mobile Firewall and intruder

The Mobile Firewall can move with the intruder and isolate the intruder for ever. Because of the dynamic topology of mobile ad hoc network, each node may move with freedom. The intruder may move out of the ring of encirclement of block agents. In order to isolate the intruder at any time, the firewall must be mobile. The Mobile Firewall is composed of two layers, block layer and defensive layer. The block layer is made up of neighbor nodes of intruder, which deter intrusion directly. The defensive layer is made up of the nodes which are outside the block layer.

Figure 2 shows that the Mobile firewall is made of two layers. Mobile ad hoc network is composed of 23 nodes in Fig 2. Node X is intruder. Nodes S, T, U, W con- 
struct the block layer of the Mobile Firewall, and they cut off the link between them and intruder X. Node J,K,M,O,P,Q,I,R construct the defensive layer of the Mobile Firewall. These nodes of the defensive layer are outside of the block layer and they are used to prevent intruder from escaping. They can not take effect when they are not neighbor of the intruder. If the intruder moves, some nodes of defensive layer may become the neighbor of the intruder. Just as figure 3, when the intruder gets at a new location, node $\mathrm{K}$ and node $\mathrm{L}$ becomes the neighbor the intruder. At that time, node $\mathrm{K}$ and node $\mathrm{L}$ change from defensive node to block node and cut off their link between them and intruder. Node B,C,D become the defensive node because they is outside of the block layer. Node U,W change from block node to defensive node when they are not neighbor of intruder. Node O,P,Q,I become common nodes. Figure 3 shows that the new Mobile Firewall is formed when the intruder moves.

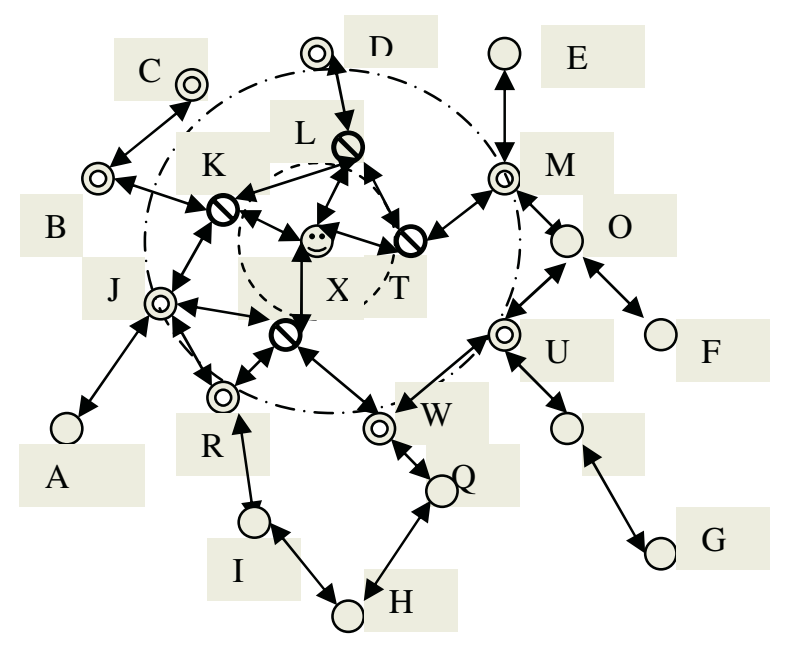

Fig. 3. The Mobile Firewall after intruder moved

In order to save network resource, the mobile firewall need not exist for ever. If the intruder stops attacking, the mobile firewall will terminate after a period. But if intruder continue to attack, the mobile firewall will keep living.

\subsection{Shrapnel Way to Dispose}

When the intruder is found, the decision agent produces block agents. The block agents get to the neighbour of the intruder and construct the Mobile Firewall to isolate the intruder. There may be some distance between the decision agent and intruder. If the decision agent produces a lot of agents and they all move from the decision agent to intruder, it will spend network bandwidth. In order to save network resource, we present the shrapnel way for agent movement. An artillery shell containing metal balls fused to explode in the air above enemy troops. By analogy, agent moves to the node 
around the intruder and reproduces a lot of agents to surround and isolate the intruder in the end.

\subsection{Local Repair}

When the intruder is found and the Mobile Firewall is built, the routes that include the intruder will break for the intruder can not receive and forward any packet. At that time the process of local repair starts to reroute the path to round the intruder. To repair the path, the node upstream of the intruder broadcasts a Route Discover packet for that destination. If, at the end of the discovery period, the repairing node has not received a Route Reply for that destination, it transmits Route Error to the originating node. The originating node may restart a process of route discover to find a fresh route to the destination. On the other hand, if the node receives one or more Route Reply packets during the discovery period, it first compares the hop count of the new route with the value and chooses a shortest path to replace the current path.

\section{Conclusion}

This paper presents the architecture of automated intrusion response which is composed of multi-agent. Monitor agents collect the information of each node and send them to decision agents. Decision agents collect the information from the monitor agent and make a judgment by security policies. If the decision agent can judge that some node is invader, it will produce block agents. These block agents move and dispose at the neighbour nodes of the intruder by shrapnel way. The Mobile Firewall is constructed to surround and isolate the intruder in the end. The Mobile Firewall can transfer when the intruder moves and will expire if the intrusion terminated. The whole response process is automated without manual intervention.

The method of intrusion detection in our architecture is too simple to detect some complex attacks. We are doing some work about intrusion detection based finite state machine [15]. We are going to use Coloured Petri Nets [16] to analyze the routing protocol and propose the method of intrusion detection.

\section{References}

1. D. Schnackenberg, H. Holliday, R. Smith, K. Djahandari, and D. Sterne, Cooperative Intrusion Traceback and Response Architecture (CITRA), Proceedings of the Second DARPA Information Survivability Conference and Exposition (DISCEXII), Anaheim, CA, June 2001.

2. D. Schnackenberg, K. Djahandari, and D. Sterne, Infrastructure for Intrusion Detection and Response, Proceedings of the DARPA Information Survivability Conference and Exposition, Hilton Head, SC, January 2000.

3. Dan Sterne, Kelly Djahandari, Brett Wilson, Bill Babson,Dan Schnackenberg, Harley Holliday, and Travis Reid, Autonomic Response to Distributed Denial of Service Attacks, In Proceedings of the 4th International Symposium on Recent Advances in Intrusion Detection, RAID 2001, Davis, CA, USA, October 2001, Springer-Verlag, pp.134-149 
4. X. Wang, D. Reeves, and S. F. Wu, Tracing-Based Active Intrusion Response, Journal of Information Warfare, Vol. 1, No. 1, September, 2001

5. N. Foukia, S. Hassas, S. Fenet and J. Hulaas, An Intrusion Response Scheme: Tracking the Source Using the Stigmergy Paradigm, in proceedings of Security of Mobile Multiagent Systems Workshop (SEMAS-2002), Bologna, Italy, July 162002

6. CA Carver, JMD Hill, JR Surdu, and UW Pooch, A Methodology for Using Intelligent Agents to Provide Automated Intrusion Response, proceedings of the 2000 IEEE workshop on Information Assurance and Security, West Point, NY,USA, June 2000.

7. Jansen, W. A. Karygiannis, T. Marks, D. G. Mell, P. M., Mobile Agents in Intrusion Detection and Response, Canadian Information Technology Security Symposium , June 1923,2000, Ottawa, Canada

8. Srdjan Capkun, Levente Nuttyan, Jean-Pierre Hubaux, Self-organized public-key Management for mobile ad hoc networks, IEEE Transactions on mobile computing, Vol.2, No.1, January-March, 2003

9. Lidong Zhou, Zygmunt J. Haas, Securing ad hoc networks, IEEE Networks Special Issue on Network Security, November/December, 1999

10. P.Papadimitratos, Z.Haas, Secure routing for mobile ad hoc networks, in Proceedings of the SCS communication Networks and Distributed Systems Modeling and Simulation Conference, San Antonio, TX, January 27-31,2002

11. Yih-Chun Hu, Adrian Perrig, David B. Johnson. Ariadne: A secure On-Demand Routing Protocol for Ad hoc Networks, in Proceedings of the MobiCom 2002, September 23-28, 2002, Atlanta, Georgia, USA

12. Kimaya Sanzgiri, Bridget Dahill, Brian Neil Levine, Clay Shields, Elizabeth M. BeldingRoyer. A Secure Routing Protocol for Ad Hoc Networks, In Proceedings of 2002 IEEE International Conference on Network Protocols (ICNP), November 2002

13. Yih-Chun Hu, David B. Johnson, and Adrian Perrig, SEAD: Secure Efficient Distance Vector Routing for Mobile Wireless Ad Hoc Networks, in Proceedings of the 4th IEEE Workshop on Mobile Computing Systems \& Applications (WMCSA 2002), pp. 3-13, IEEE, Calicoon, NY, June 2002

14. Yongguang Zhang \& Wenke Lee, Intrusion Detection Techniques for Mobile Wireless Networks, Mobile Networks and Applications, 2003

15. Ping Yi, Yichuan Jiang, Yiping Zhong, Shiyong Zhang, Distributed Intrusion Detection for mobile ad hoc networks, The 2005 International Symposium on Applications and the Internet(SAINT2005), Trento, Italy, January 31 - February 4, 2005

16. S. Gordon, L. Kristensen and J. Billington. Verification of a Revised WAP Wireless Transaction Protocol. In Application and Theory of Petri Nets 2002, vol. LNCS 2360, pages 182-202, 2002. Springer Verlag, Berlin Heidelberg New York. ISBN 3-540-437878. Presented at the 23rd International Conference on Application and Theory of Petri Nets, Adelaide, Australia, 24-30 Jun 2002. 